\title{
Spotlight on atezolizumab and its potential in the treatment of advanced urothelial bladder cancer
}

\author{
This article was published in the following Dove Press journal: \\ OncoTargets and Therapy \\ 9 March 2017 \\ Number of times this article has been viewed
}

\author{
Ahmet Murat Aydin $1, *$ \\ Solomon L Woldu',** \\ Ryan C Hutchinson' \\ Martin Boegemann ${ }^{2}$ \\ Aditya Bagrodia' \\ Yair Lotan' \\ Vitaly Margulis' \\ Laura-Maria Krabbe ${ }^{1,2}$ \\ 'Department of Urology, University \\ of Texas Southwestern Medical \\ Center, Dallas, TX, USA; ${ }^{2}$ Department \\ of Urology, University of Münster \\ Medical Center, Münster, Germany \\ *These authors contributed equally \\ to this work
}

\begin{abstract}
Metastatic urothelial carcinoma of the bladder is an aggressive malignancy with poor prognosis, reflecting a lack of effective systemic therapies. The current standard of care includes multiagent platinum-based chemotherapy; however a majority of patients do not respond to treatment and most eventually succumb to disease. Recently, renewed interest in immunotherapy in the form of immune-checkpoint inhibition has gained widespread attention for a number of malignancies. Atezolizumab, an anti-PDL1 antibody, has been shown to be effective in a subset of patients previously treated with or unfit for platinum-based chemotherapy, and has shown durable responses with a good tolerability profile. We review the mechanism of action and clinical evidence of atezolizumab for metastatic urothelial bladder cancer, and discuss this drug within the context of ongoing developments in this dynamic field of immunooncology.
\end{abstract}

Keywords: atezolizumab, MPDL3280A, bladder cancer, PDL1, immunotherapy, metastatic

\section{Introduction}

Bladder cancer is the ninth-commonest malignancy worldwide, with differences in prevalence explained largely by differences in exposure to certain risk factors. ${ }^{1,2}$ In the developed world, where the main risk factor is tobacco exposure, the vast majority of bladder cancer is of urothelial histology. ${ }^{1,3}$ In other regions, where Schistosoma haematobium infection is common, squamous cell carcinoma histology is predominantly present. ${ }^{4}$ While most urothelial carcinoma of the bladder (UCB) presents as non-muscle-invasive disease, approximately $30 \%$ of bladder cancers are already muscle-invasive at diagnosis. ${ }^{5}$ When amenable to surgical therapy, approximately $25 \%$ of patients with muscle-invasive disease are found to harbor lymph-node metastasis, with associated decreased survival due to the frequent development of distant metastases. Additionally, about $10 \%$ of patients present with systemic metastatic disease at the time of diagnosis. ${ }^{6}$

Since the 1970s, the cornerstone of therapy for advanced and metastatic UCB (mUCB) has been platinum-based chemotherapy (PBCT), first as a single agent and subsequently as part of combination therapy. ${ }^{7}$ Since the 1990 s, the standard first-line therapy has been methotrexate, vinblastine, adriamycin, and cisplatin (MVAC) after demonstrating a superior response rate and overall survival (OS) compared to cisplatin alone. ${ }^{8}$ A number of chemotherapeutic regimens have been evaluated to improve the response rate and significant toxicity profile of $\mathrm{MVAC}$, with gemcitabine and cisplatin showing similar overall response rate and OS with a better tolerability profile. ${ }^{9}$ As such, both MVAC and gemcitabine-cisplatin are considered first-line therapies for mUCB. Numerous permutations of these platinum-containing regimens have been studied, including high-intensity MVAC, coadministration of growth-factor support, and substitution of other platinum agents (eg, carboplatin) for those unable to receive
Correspondence: Laura-Maria Krabbe Department of Urology, University of Texas Southwestern Medical Center, 5323 Harry Hines Boulevard - J8. I24, Dallas, TX 75390-91 10, USA

$\mathrm{Tel}+\mathrm{I} 2146480567$

Fax + I 2146488786

Email laura-maria.krabbe@ utsouthwestern.edu (c) (1) (5) 2017 Aydin et al. This work is published and licensed by Dove Medical Press Limited. The full terms of this license are available at https://www.dovepress.com/terms.php cc. ${ }_{\mathrm{BY}} \mathrm{NC}$ and incorporate the Creative Commons Attribution - Non Commercial (unported, v3.0) License (http://creativecommons.org//icenses/by-nc/3.0/). By accessing the work you hereby accept the Terms. Non-commercial uses of the work are permitted without any further permission from Dove Medical Press Limited, provided the work is properly attributed. For permission for commercial use of this work, please see paragraphs 4.2 and 5 of our Terms (https://www.dovepress.com/terms.php). 
cisplatin. ${ }^{7}$ While advances in systemic chemotherapies have led to modest improvements in outcomes, mUCB remains a deadly disease in the majority of cases, with median progression-free survival (PFS) and OS at approximately 8 and 14 months, respectively. ${ }^{7}$

Options for patients who have progressed after first-line therapy have historically been limited. Only vinflunine monotherapy has been proven to be superior to best supportive care (BSC) alone, demonstrating a modest survival benefit (6.9 vs 4.3 months, hazard ratio $0.78,95 \%$ confidence interval [CI] $0.61-0.96 ; P=0.0227$ ) in the eligible population, which was defined as patients without major protocol violations. ${ }^{10}$ In the intention-to-treat population, the survival benefit was not statistically significant. ${ }^{10}$ While this drug was approved as a second-line agent in Europe, until recently there has not been a US Food and Drug Administration (FDA)-approved regimen for second-line use in mUCB. ${ }^{11}$

Recently, a Phase II clinical trial was conducted to evaluate an anti-PDL1 monoclonal antibody - atezolizumab (MPDL3280A; Hoffman-La Roche, Basel, Switzerland) - for treatment of mUCB after failure of PBCT or in platinumineligible patients. The findings demonstrated an overall response rate of $15 \%$ in the entire study population, a favorable tolerability profile, and long lasting responses not seen in mUCB to that point. ${ }^{12}$ These encouraging findings led to the granting of guaranteed breakthrough status and further early
FDA approval in 2016; the first new drug approved for mUCB in over 20 years. In this manuscript, we review the mechanism of action and clinical evidence of efficacy of atezolizumab for mUCB, and discuss this drug within the context of ongoing developments in this dynamic field of immunooncology.

\section{Mechanism of action Immunooncology}

In the last few years, we have seen a great deal of excitement about co-opting the immune system to fight malignancies through approaches with monoclonal antibodies, cancer vaccines, and cytokine therapies. ${ }^{13}$ One of the most attractive modalities is the activation of antitumor activity by blocking immune checkpoints (ICPs). ${ }^{14}$ The numerous genetic and epigenetic alterations that characterize malignant cells are thought to result in antigens that could reasonably be used to distinguish these cells from benign counterparts. The concept of immunosurveillance is based on this premise: circulating immune cells can attack and destroy premalignant or malignant cells, in a similar way to how these cells might act against infectious pathogens. However, immunoactivation is a complex phenomenon, requiring an intricate balance between stimulatory and inhibitory signals. ${ }^{15}$ To prevent autoimmunity, ICPs (inhibitory signals) are crucial, and their expression can be altered by tumors; an important mechanism of immune resistance (Figure 1). Studies indicate that



Figure I Mechanism of anti-PDI and anti-PDLI checkpoint blockades.

Notes: PDI is expressed by T cells. PDLI is expressed in tumor cells and tumor-infiltrating immune cells. Combination of PDI and PDLI/PDL2 contributes to the suppression of T-cell function. Inhibiting the interaction of PDI and its ligands can significantly enhance T-cell function, resulting in antitumor activity. Reprinted from Cancer Treat Rev, 4I(10), Meng X, Huang Z, Teng F, Xing L, Yu J, Predictive biomarkers in PD-I/PD-LI checkpoint blockade immunotherapy, 868-876, Copyright (20I5), with permission from Elsevier. ${ }^{94}$ 
blocking these ICPs results in an increase in effector T cells and inflammatory cytokines within the tumor, as well as a decrease in immunoregulatory cells. ${ }^{16}$

\section{Immune-checkpoint inhibition}

Inhibition of these ICPs to alter the tumor microenvironment is one of the most promising areas of active cancer research. T-cell-receptor activation requires costimulatory molecules and the absence of inhibitory molecules. The first ICP inhibitor (ICPI) clinically investigated was ipilimumab (MDX-010; Bristol-Meyers Squibb, New York, NY, USA), a monoclonal anti-CTLA4 antibody that blocks the interaction of B7 (a costimulatory molecule on antigen-presenting cells) with the inhibitory CTLA4 receptor expressed on the surface of T cells. ${ }^{17}$ Ipilimumab has been shown to be effective in metastatic melanoma, and gained FDA approval in $2011 .^{18}$ One drawback of this approach appears to be a lack of selectivity of CTLA4 blockade in T-cell expansion, and this may underlie the significant immunorelated toxicities associated with ipilimumab. ${ }^{19}$

Another ICP is the interaction between PD1 and its ligands PDL1 and PDL2. A more exhaustive review of this ICP is available by Ohaegbulam et al. ${ }^{20}$ Briefly, PD1 is expressed on a number of immune cells, including activated $\mathrm{T}$ cells, regulatory $\mathrm{T}$ cells, $\mathrm{B}$ cells, monocytes, natural killer cells, and dendritic cells. For activated T cells, binding of PD1 by its ligands results in inhibition of the T-cell receptor and subsequent termination of the immune response. This is an important physiological mechanism to avoid autoimmunity; however, when expressed by tumor cells (TCs) or tumor-infiltrating immune cells (ICs) within the tumor microenvironment, this leads to termination of the antitumor activity of T cells in an analog fashion..$^{20,21}$ Tumors themselves can express these inhibitory ligands, as well as induce their expression by immune-infiltrating cells, resulting in an effective checkpoint against immunosurveillance..$^{20,22}$ Cancer therapeutics in the form of monoclonal antibodies that block the interaction between the PD1 receptor and its ligands have been a very active area of research, with promising results in a number of malignancies.

A number of monoclonal antibodies have been developed to target PD1, including pidilizumab (CT-011; CureTech, Yavne, Israel), ${ }^{23}$ pembrolizumab (MK-3475; Merck, Kenilworth, NJ, USA), ${ }^{24}$ and nivolumab (BMS-936558; Bristol-Meyers Squibb). ${ }^{25}$ The most extensively studied of these is nivolumab, which has been FDA-approved in non-small-cell lung cancer, Hodgkin lymphoma, metastatic melanoma, and metastatic renal cell carcinoma. ${ }^{26}$ Of the two ligands that are known to activate PD1, PDL1 appears to play a more prominent role in lymphocyte regulation, ${ }^{27}$ and as such a number of agents have been developed to block PDL1, including atezolizumab, ${ }^{28}$ BMS-936559 (BristolMeyers Squibb), ${ }^{29}$ durvalumab (MEDI4736; AstraZeneca, London, UK), ${ }^{30}$ and avelumab (MSB0010718C; Pfizer, New York, NY, USA). ${ }^{31}$ These agents are the subjects of ongoing clinical trials.

\section{Immunotherapy in bladder cancer}

While there is a growing interest in immunotherapies for systemic malignancies, immunotherapy actually has an extensive history in bladder cancer, albeit for non-muscle-invasive disease. Forty years ago, Morales et al demonstrated that intravesical instillation of bacillus Calmette-Guérin (BCG), an attenuated strain of Mycobacterium bovis, was effective at treating noninvasive bladder cancer, ${ }^{32}$ and this form of immunotherapy remains the standard of care today for high-risk non-muscle-invasive disease. ${ }^{33}$ The mechanism underlying the efficacy of BCG remains incompletely elucidated, but it is broadly believed that BCG activates the immune system and induces an inflammatory response, ultimately leading to immunomediated cytotoxicity through $\mathrm{CD}^{+} \mathrm{T}$ lymphocytes, natural killer cells, and granulocytes. ${ }^{34}$

This evidence of effective immunomanipulation in the treatment of noninvasive disease and growing evidence of efficacy of immunotherapies, in particular ICP inhibition, in the treatment of other nonlocalized malignancies led to the logical investigation of ICP inhibition as a potential therapy for mUCB. Inman et al described PDL1 TC expression by immunohistochemistry (IHC) in 280 high-risk bladder patients, and found that PDL1 expression increased significantly with higher disease stage and grade, hypothesizing that PDL1 expression may be one mechanism of BCG resistance. ${ }^{35}$ Additionally, a hallmark of UCB is the presence of a high somatic mutational load, ${ }^{36}$ which may result in increased neoantigen expression and may make the tumors more susceptible to immunosurveillance. ${ }^{13}$

\section{Atezolizumab}

Pharmacokinetics and pharmacodynamics

Atezolizumab (MPDL3280A) was developed as a human monoclonal $\mathrm{IgG}_{1}$ antibody with a high affinity for PDL1 (binding affinity dissociation constant $=0.4 \mathrm{nM}$ ). ${ }^{37}$ Atezolizumab blocks the interaction of PDL1 (also called B7-H1 or CD274) with PD1 and B7.1 (also called CD80). ${ }^{37,38}$ Both PD1 and B7.1 are receptors for PDL1 binding, which results in T-cell tolerance and restriction of cellular killing. Therefore, the inhibition of the host immune response against the tumor can be prevented by interaction of the anti-PDL1 antibody 
with its ligand. Of note, atezolizumab has an engineered fragment $(\mathrm{Fc})$ domain that prevents active T-cell depletion via antibody-dependent cellular toxicity.

It has a half-life of 27 days, and steady-state concentration is reached in two to three cycles (6-9 weeks) of repeated intravenous (IV) doses..$^{39}$ Its volume of distribution is $6.9 \mathrm{~L}$ and clearance is $0.2 \mathrm{~L} /$ day. Systemic accumulation area under the curve (AUC), maximum concentration $\left(\mathrm{C}_{\max }\right)$, and minimum concentration $\left(\mathrm{C}_{\text {min }}\right)$ are 1.91-, 1.46-, and 2.75-fold, respectively. Atezolizumab follows a biphasic distribution until day 7 after IV bolus administration. ${ }^{40}$ It shows nonlinear (dose-dependent) and linear (dose-independent) pharmacokinetics in doses of $0.5-5 \mathrm{mg} / \mathrm{kg}$ and $5-20 \mathrm{mg} / \mathrm{kg}$, respectively. Herbst et al detected antitumor activity in doses of $1-20 \mathrm{mg} / \mathrm{kg}$ following IV administration once every 3 weeks. ${ }^{37}$

\section{Available data for atezolizumab in $\mathrm{mUCB}$}

Most initial studies evaluating the efficacy and safety of antiPDL1 treatments were based on biomarker-enriched cohorts, including only patients with positive immunoreactivity for PDL1. However, Powles et al expanded the initial cohort in their Phase I multicenter dose-escalation expansion study (NCT01375842/PCD4989g) to mUCB patients without immunoreactivity against PDL1. ${ }^{28}$ Patients were scored according to IHC status within a range between 0 and 3 . Among a total of 67 enrolled patients, $18 \%$ had no expression of PDL1 (IHC score 0), 34\% low expression (IHC score 1), $30 \%$ intermediate expression (IHC score 2), and 15\% high expression (IHC score 3). Most patients (93\%) had received previous $\mathrm{PBCT}$, and $72 \%$ had received two or more lines of treatment. Patients with an Eastern Cooperative Oncology Group (ECOG) score $\geq 2$ were excluded. Patients received atezolizumab at a dose of $15 \mathrm{mg} / \mathrm{kg}$ IV every 3 weeks. The first infusion of atezolizumab lasted 60 minutes, which was subsequently reduced to 30 minutes if well tolerated. The total number of planned cycles was 16 , comprising a total treatment time of 1 year. The reasons for termination of treatment were progression of disease according to Response Evaluation Criteria in Solid Tumors (RECIST) version 1.1 ${ }^{41}$ or immunorelated response criteria, ${ }^{42}$ intolerable toxicity, and incompliance with the study protocol. After a minimum 6 weeks of follow-up, $43 \%$ of patients with IHC score $2 / 3$ tumors and $11 \%$ of patients with IHC score $0 / 1$ tumors showed an objective response (OR; complete remission and partial remission). Of those with IHC score $2 / 3$ tumors, $7 \%$ had a complete response. Following the outcomes of this study, atezolizumab was granted breakthrough status for mUCB by the FDA. ${ }^{28}$
A subsequent Phase II study with two subgroups (group 1, patients with $\mathrm{MUCB}$ ineligible for PBCT for first-line treatment; group 2, patients with $\mathrm{mUCB}$ after progression on PBCT for second-line treatment) was performed (NCT02108652). ${ }^{43}$ In 2016, Rosenberg et al reported on group 2 of the Phase II trial (NCT02108652/IMvigor210), in which 310 patients with $\mathrm{mUC}$ whose disease had progressed despite previous treatment with PBCT were enrolled. ${ }^{12}$ The urinary bladder was the primary tumor site in $74 \%$ of patients, while the renal pelvis, ureter, urethra, and other sites were the primary site in $14 \%, 7 \%, 2 \%$, and $3 \%$ of patients, respectively.

Patients were grouped according to PDL1-expression status, which was based on percentage of PDL1-positive ICs within the tumor environment determined by the SP142 assay (Hoffman-La Roche): IC0 ( $<1 \%$ ), IC1 ( $\geq 1 \%$ but $<5 \%$ ), and IC2/3 ( $\geq 5 \%$ ). Of the 310 patients included 103, 107, and 100 were in the IC0, IC 1 , and IC2/3 groups, respectively. A standard fixed dose of $1,200 \mathrm{mg}$ was administered every 3 weeks, with a median duration of 12 (range 0-66) weeks. OR rates (ORRs) were $26 \%, 18 \%, 11 \%$, and $8 \%$ in IC groups $2 / 3,1 / 2 / 3,1$, and 0 , respectively. Therefore, a combined ORR of $15 \%$ was reported for all patients in the study. In comparison to the previously published Phase I expansion cohort, the response rate of IC $2 / 3$ patients was lower $(26 \%$ vs 43\%); however, in the Phase I cohort, ICs and TCs were evaluated for PDL1 expression, whereas in the Phase II cohort only ICs were considered.

The median time to response was about 2 months. After a median follow-up of 11.7 months, $84 \%$ of the initially responding patients showed ongoing response to therapy (median time of response not reached), including those with poor risk factors and upper-tract disease. Interestingly, many responses were ongoing, despite discontinuation of treatment for various reasons. For the entire cohort, median PFS and OS were 2.1 and 7.9 months, respectively, while for IC2/3 patients median PFS and OS were 2.1 and 11.4 months, respectively. An updated report of the same trial after approximately 1.5 years of median follow-up revealed 1-year OS rates of $50 \%$ for patients with IC $2 / 3$ and $37 \%$ for the overall cohort. ${ }^{44}$ Complete responses occurred in $7 \%$ of the overall cohort, as well as $15 \%, 6 \%$, and $2 \%$ of patients with IC2/3, IC 1 , and IC0, respectively, with median duration of response not reached. For mUC of the upper urinary tract, OS was 7.6 and 10.9 months, whereas for metastatic bladder cancer OS was 7.9 and 12.8 months, for the overall cohort and $\mathrm{IHC} 2 / 3$ patients, respectively.

Due to these favorable results, after which atezolizumab was FDA-approved for second-line therapy, it is currently being evaluated as part of a Phase III randomized clinical 
trial (IMvigor211) that is comparing atezolizumab to other chemotherapeutic agents (docetaxel, paclitaxel, vinflunine) in patients with locally advanced and MUCB who have progressed during or following PBCT. The results of this ongoing study are expected in mid-2017.

Further, regarding first-line use of atezolizumab, the data for group 1 of the phase II IMvigor trial have not been finally published, but excerpts were presented at 2016 annual cancer meetings. ${ }^{45,46}$ Overall, 119 patients were included in group 1 of IMvigor210, and the definition of platinum ineligibility was one or more of the following: glomerular filtration rate (GFR) 30-60 $\mathrm{mL} / \mathrm{min}(70 \%), \geq$ grade 2 hearing loss $(14 \%)$ /neuropathy $(6 \%)$, or ECOG performance status of $2(20 \%)$. The primary end point was ORR, and the treatment regimen was the same as in group 2. Of the whole cohort, $92 \%$ had metastatic disease (66\% visceral metastases), and the bladder was the primary tumor site in $71 \%$ of patients. Again, the patients were grouped by PDL1-expression status on ICs as described earlier, and 33\% were IC $0,40 \%$ were IC 1 , and $27 \%$ were IC2/3.

The ORR for the whole cohort was 24\% (7\% complete response and $17 \%$ partial response). Here as well, some correlation with the IC group and response to therapy was seen; however, it was not as pronounced as in group 2 . ORRs in groups IC0, IC 1 , and IC2/3 were $21 \%, 23 \%$, and $28 \%$, respectively, with all groups showing $6 \%-8 \%$ complete response. Also in this trial, responses were durable, and after a median follow-up of 14.4 months, median OS was 14.8 months (95\% CI 10.1 months to not reached) and $57 \%$ alive at 12 months. Interestingly, patients in the IC0/1 groups fared similarly to patients in the IC2/3 group (15.3 vs 12.3 months, respectively). These favorable data might lead to approval in the first-line space for non-platinum-eligible patients in the near future.

Additionally, there are other ongoing clinical trials of atezolizumab for different stages of urothelial carcinoma (UC), especially in the adjuvant setting, in which PBCT is the current standard of care, as well as for high-risk non-muscleinvasive $\mathrm{UCB}$, in which intravesical treatment, foremost with BCG, is currently utilized. ${ }^{1}$ A summary of currently listed clinical trials on ClinicalTrials.gov of atezolizumab for UC is presented in Table 1. ${ }^{12,28,37,43,45-58}$ More information regarding efficacy and safety will become available through these trials, and new indications for atezolizumab and treatmentresponsive patient subgroups may be discovered.

Biomarkers are vital in oncology to determine patients who might or might not be responsive to certain treatments. With regard to immunobiomarkers for atezolizumab, the SP142 assay was developed using rabbit monoclonal
anti-PDL1 clone SP142 for assessment of PDL1 expression in UC and non-small-cell lung carcinoma. ${ }^{37}$ The IHC assay was performed in formalin-fixed, paraffin-embedded urothelial tissue and based on the percentage of TCs or ICs with PDL1 expression, regardless of intensity. Both tumors and ICs showed membranous and cytoplasmic staining; however, detection of PDL1 expression was difficult in ICs, which had scant cytoplasms and were small. Distribution of PDL1-expressing TCs was typically focal, and mostly located at the interface between malignant cells and stroma. However, distribution and location of PDL1-expressing ICs varied within tumors; they were located at either the periphery of the tumor, at stromal bands dissecting the tumor, within IC aggregates, or scattered as single cells throughout. IHC status of specimens was categorized into four groups: IHC0, $-1,-2$, or -3 if the percentage of PDL1-positive cells per area was $<1 \%, \geq 1 \%$ but $<5 \%, \geq 5 \%$ but $<10 \%$, or $\geq 10 \%$; respectively. Patients with multiple specimens were scored according to the specimen with the highest score.

Rosenberg et al determined a cutoff value of $\geq 5 \%$ PDL1 expression in ICs determined by SP142 assay as high, which was associated with response. ${ }^{12}$ However, although PDL1 positivity was beneficial for response to treatment, ORRs of approximately $10 \%$ were also detected in PDL1-negative patients with mUCB. In addition to PDL1-expression status, mutation load and Cancer Genome Atlas gene expression were analyzed with respect to PDL1 expression, as well as response. Despite significantly higher prevalence of PDL1 positivity in the basal vs the luminal subtypes of UC $(60 \%$ vs 23\%), the highest response rate was seen in cluster II of the luminal subtype (34\%). The authors concluded that future multibiomarker systems might accurately predict response to atezolizumab. Bellesoeur et al evaluated 346 patients with various malignancies eligible for treatment with ICPI, and found $\mathrm{UC}$ was associated with higher PDL1 expression than other tumor types. ${ }^{59}$ However, depending on the assay used for PDL1 evaluation, as well as the cell type studied, PDL1 expression can vary. ${ }^{28}$

In many solid cancers, OR is better correlated with PDL1 expression on TCs than on $\mathrm{ICs}^{60}{ }^{60}$ contrary to $\mathrm{mUCB}$, in which PDL1 expression on ICs is favorable. PDL1 expression is a dynamic process, requires recognition of antigens by ICs, and might differ during course of treatment, ${ }^{61}$ and thus subsequent examinations of IHC status may be necessary after initial evaluation prior to treatment. In malignant melanoma and renal cell carcinoma, heterogeneity of PDL1 expression has been reported between primary tumor and metastatic sites. ${ }^{62,63}$ 


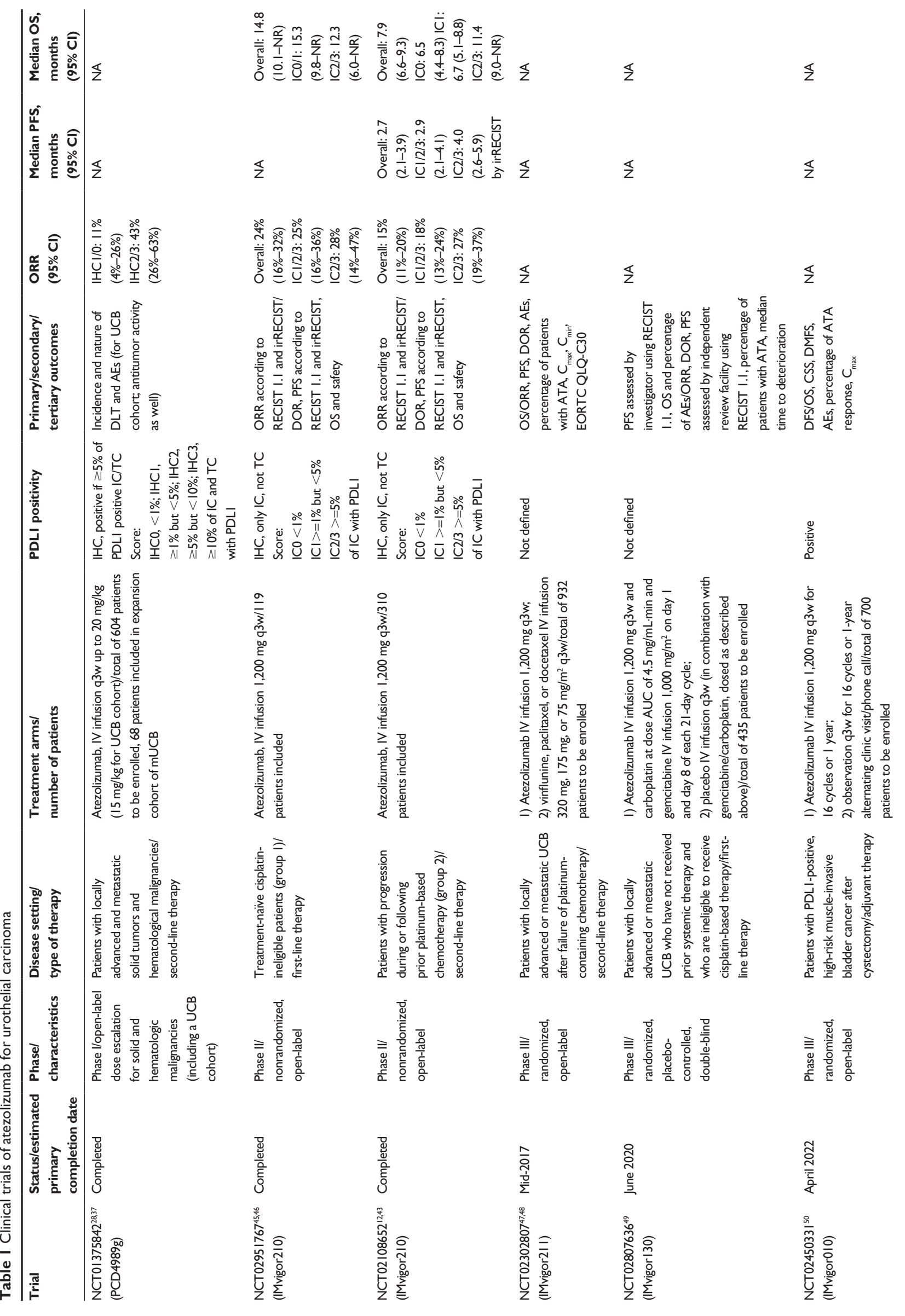




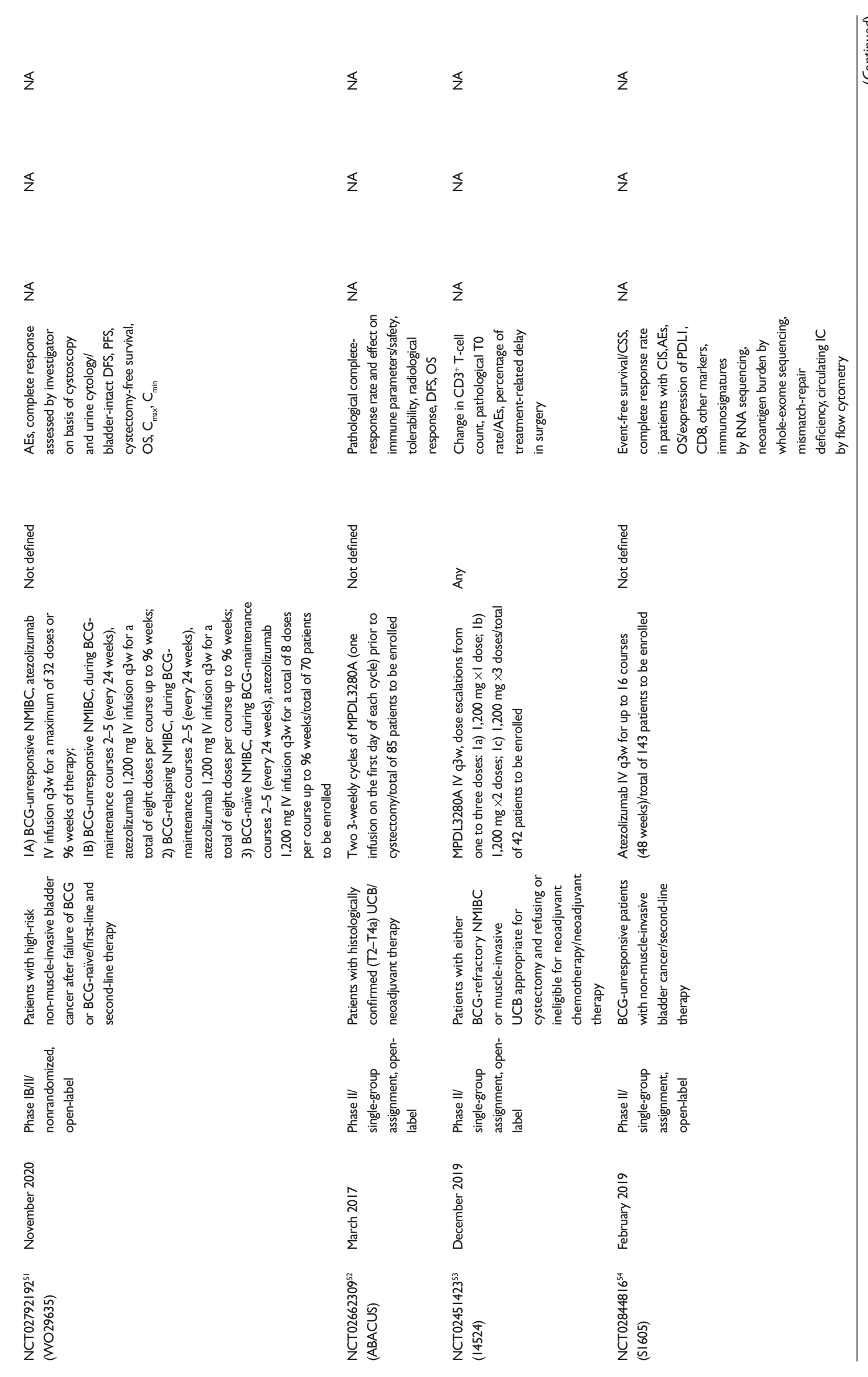




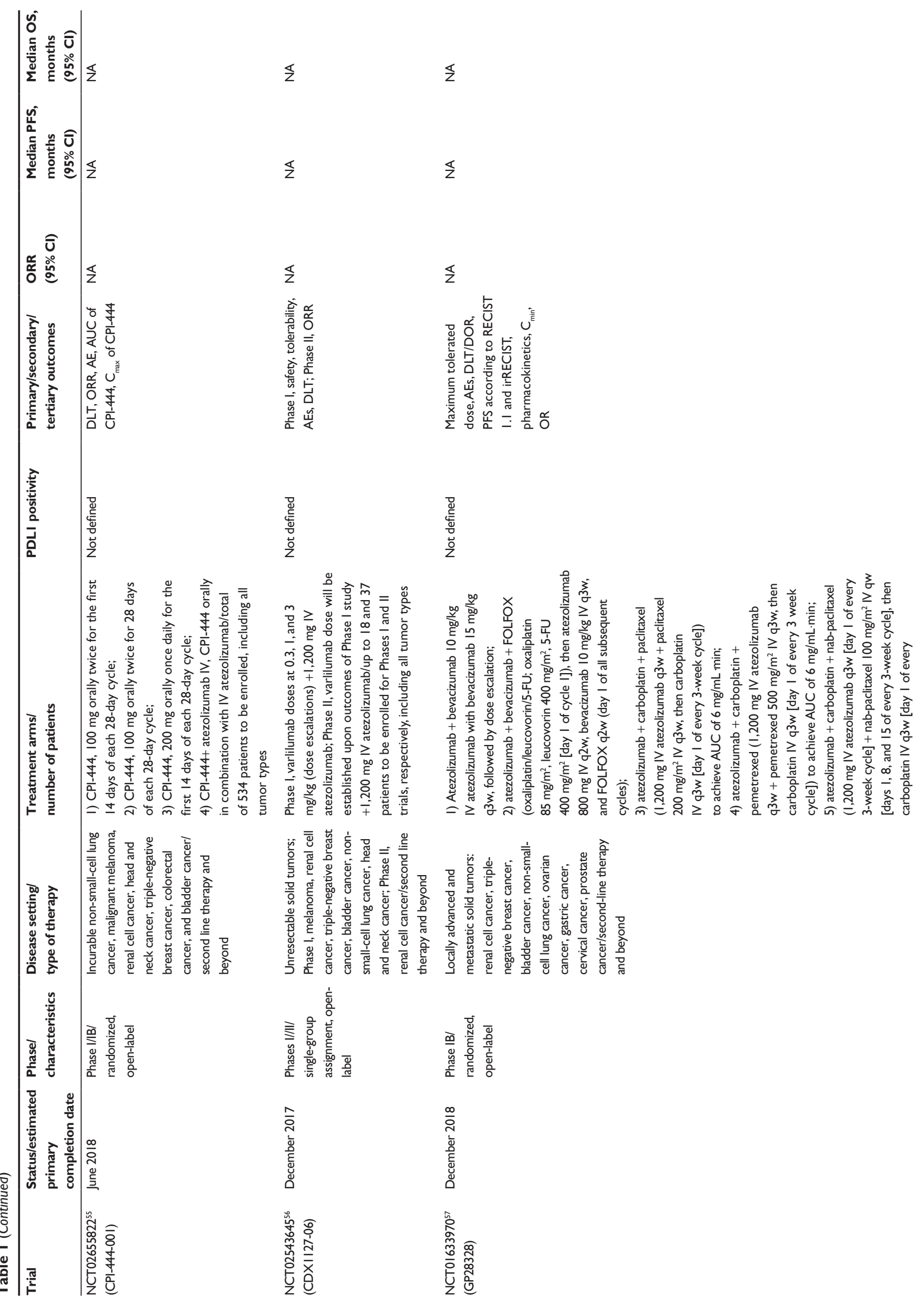




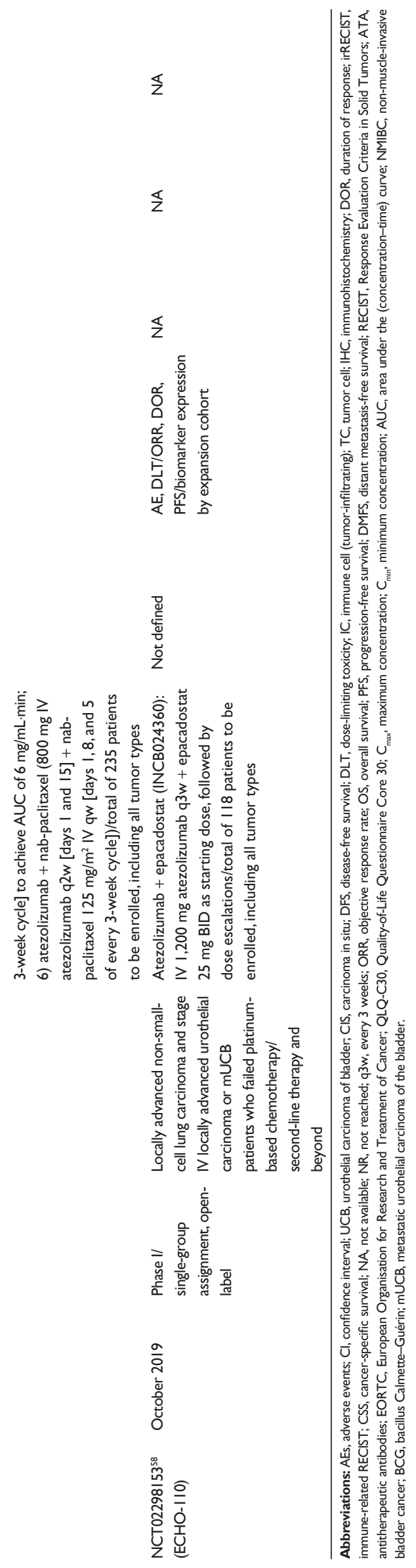

There are still limitations with such biomarkers as PDL1 expression for predicting response to treatment. These limitations are due to the multitude of PDL1 antibodies, assays, scoring systems, and thresholds for positivity. However, there is still value in knowing the PDL1 expression, since in the Rosenberg et al study ORRs were 26\% (95\% CI 18\%-36\%) in the combined IC2 and IC3 group and only $10 \%$ and $8 \%$ in the IC1 and IC0 groups, respectively. ${ }^{12}$ At this time, due to limitations in understanding the role of a biomarker in selecting patients for therapy, the FDA does not require PDL1 positivity for initiation of atezolizumab treatment for mUCB patients.

Blood-based immunobiomarkers, such as IL-6, IL-18, and IFN $\gamma$, have not been found to be associated with response to atezolizumab treatment. ${ }^{37}$ Anantharaman et al evaluated the value of circulating TCs (CTCs) with PDL1 expression as a noninvasive and safe biomarker in 25 patients with muscleinvasive bladder cancer or mUCB. ${ }^{64}$ While CTCs were found in $80 \%$ of patients overall, $35 \%$ of CTCs showed PDL1 positivity. Although statistically insignificant as the study was not powered to detect signals for survival, patients with a high PDL1-positive CTC burden showed decreased median OS (194 days) compared to those with low PDL1-positive CTC burden (303 days). Of note, most PDL1-positive CTCs showed no cytokeratin expression in the analysis, which might be a sign of epithelial-mesenchymal transition during metastasis development and consistent with escape from immunosurveillance by PDL1 expression. However, only five patients had anti-PDL1 therapy after analysis of CTCs, thus limiting the value of the study regarding predictive information. Moreover, in non-small-cell lung cancers, certain types of blood cells, which showed PDL1 expression without expression of common myeloid specific markers, resulted in decreased specificity and interfered with identification of true TCs in CTC analysis, thus indicating possible problems with this approach. ${ }^{65}$

Theoretically, a multitude of possibilities regarding biomarkers are possible, including CTCs, cell-free DNA, microRNA, specific tumor mutations, or number of mutations, as well as markers of immunoactivity; however, reliable data in this regard is still lacking. Finding and improving biomarkers, as well as assays and cutoff values, may enable better selection of patients for more accurate prediction of treatment outcomes, while reducing toxicity and cost.

Furthermore, pseudoprogression or atypical response patterns have been described in patients receiving immunotherapy for solid tumors. ${ }^{42}$ Patients may have favorable response, despite an initial increase in tumor size or even appearance of new lesions during immunotherapy, possibly due to increased 
IC localization and inflammation in tumor sites. Therefore, contrary to cytotoxic agents, RECIST 1.1 is not always an appropriate tool for evaluation of response to immunotherapeutic agents. Loriot et al reported that among mUCB patients who continued atezolizumab despite progression of disease, $19 \%$ had a subsequent $\geq 30 \%$ decrease in target lesions from their baseline scans, thus warranting reliable biomarkers to determine treatment success, as well as innovative methods to differentiate late response and primary failure. ${ }^{44}$

As late favorable response to atezolizumab treatment occurs in some patients, when to initiate systemic therapy after failed salvage immunotherapy remains an open question. Furthermore, management of patients with mUCB who have failed or had to discontinue salvage anti-PD1/PDL1 immunotherapy following failed first-line systemic therapy is not yet defined. Nevertheless, Sonpavde et al reported that the median survival rate of these patients was comparable (189 days after start of anti-PD1/PDL1 therapy) to historically observed patients who received second-line systemic therapy only after PBCT. ${ }^{66,67}$ The only significant prognostic factor for survival was patient performance status. Response to and duration of prior immunotherapy was not correlated with survival. Therefore, delivery of third-line and beyond therapies for patients who have failed salvage anti-PD1/PDL1 immunotherapy seems possible.

\section{Alternative agents to atezolizumab for second-line therapy for $\mathrm{mUCB}$}

The prognosis of patients with mUCB who fail PBCT is very poor, and prior to the approval of atezolizumab, no secondline therapy for mUCB was available in the US, whereas vinflunine was available only in Europe and showed limited efficacy. So far, very few randomized clinical trials have been performed in the second-line setting for $\mathrm{mUCB}$, as trials have been limited by poor performance status, impaired renal function, and comorbidities that restricted trial design and contributed to poor patient accrual. ${ }^{47}$ Furthermore, significant disease heterogeneity exists, which limited the interpretation and applicability of Phase II trials.

While no data comparing atezolizumab with other treatments are available yet, the published results for atezolizumab exceed the ones of previously evaluated second-line agents. Studies evaluating the impact of rechallenging with MVAC reported an ORR of approximately $20 \%$ in mUCB patients. ${ }^{68,69}$ Several monotherapies and combinations have been utilized in patients who progressed after first-line platinum-based regimens. The highest ORRs reported for docetaxel, gemcitabine, ifosfamide, fluorouracil/recombinant human IFN $\alpha_{2 a}$, paclitaxel, and pemetrexed monotherapy as second-line therapy were $6 \%$ (44\% for patients with subsequent platinumcontaining chemotherapy), $11 \%, 20 \%, 30 \%, 10 \%$, and $27.7 \%$, respectively..$^{70-75}$ However, these Phase II trials were usually hampered by a low number of patients (generally ten to 30), methodological issues (no randomization or no blinding), poor study designs (selection bias and no prior stratification of risks), and associated with serious adverse events (AEs), such as grade 3/4 myelosuppression and neuropathy. Research evaluating oxaliplatin, piritrexim, and irinotecan as secondline therapy for this setting also reported poor outcomes and was hampered by similar limiting factors. ${ }^{76}$ Sorafenib, aflibercept, and pazopanib as second-line targeted therapy in UC demonstrated ORRs of $0,5 \%$, and $17 \%$, respectively, with a high rate of AEs. ${ }^{77-79}$ The trial in which the combination of everolimus with paclitaxel was studied was negative as well. ${ }^{80}$ None of these agents received approval for second-line therapy, due to their limited efficacy and high toxicity.

To our knowledge, other than atezolizumab, there are only two completed Phase III trials for second-line therapy of mUCB. The first was a report in 2009 from the Phase III trial of vinflunine, a second-generation vinca-alkaloid, which inhibits microtubules (NCT00315237). ${ }^{67}$ The study was designed to compare OS of UC patients receiving second-line vinflunine and $\mathrm{BSC}$ to patients receiving $\mathrm{BSC}$ only (palliative radiotherapy, antibiotics, analgesics, corticosteroids, and transfusions) after failure of PBCT. A total of 253 patients were randomly assigned to vinflunine plus $\mathrm{BSC}$, while 117 patients received BSC only. Of note, some patients received vinflunine with dose reductions, due to serious hematologic AEs observed during the trial. Overall response rate ( $8.6 \%$ vs $0 \%$ ) and median PFS (3 vs 1.5 months) were all statistically significant in favoring the treatment arm. In the vinflunine-treatment arm, approximately half of patients had grade 3/4 neutropenia, whereas grade 3/4 levels of febrile neutropenia, anemia, fatigue, and constipation were observed in $6 \%, 19 \%, 19 \%$, and $16 \%$ of patients, respectively. The updated study with a median follow-up of 45.4 months reported that vinflunine increased survival by 2.3 months and provided a partial response rate of $10 \%{ }^{10}$ After these results, it was approved in Europe and European Association of Urology guidelines recommended vinflunine as a second-line agent in mUCB patients who failed PBCT; however, as mentioned earlier, statistical significance was lacking in the intention-to-treat cohort, which probably precluded approval by the FDA. ${ }^{81}$

The other Phase III trial for advanced UC evaluated the combination of gemcitabine and paclitaxel as second-line 
therapy (German Cancer Society/Deutsche Krebsgesellschaft Studien ID 213, Studienkennung 01-09). ${ }^{82}$ All patients received 3-week cycles of gemcitabine and paclitaxel until either a maximum of six cycles (48 patients, short-term treatment arm) or until documented disease progression (48 patients, prolonged-treatment arm). Both OS (7.8 vs 8 months) and PFS rates (4 vs 3.1 months) were comparable. Furthermore, patients experienced severe anemia, two patients died due to treatment-related AEs (TrAEs; pulmonary fibrosis and neutropenic septicemia), and six patients were withdrawn from the prolonged treatment due to disease progression and toxicity. Therefore, it was concluded that the prolonged combined regimen of gemcitabine and paclitaxel for $\mathrm{mUCB}$ in second-line settings was not feasible.

Atezolizumab as second-line/salvage therapy for patients who progressed during or after PBCT had comparable OR and OS rates to other agents. Moreover, as described earlier, atezolizumab treatment is associated with durable response, has a favorable safety profile with low incidence of grade 3-5 AEs, and does not necessitate any dose adjustments for the majority of comorbidities, as it allows for treatment in spheres that cytotoxic agents do not; therefore, it seems the best choice for second-line therapy of $\mathrm{mUC}$ in the light of currently available literature.

In addition to atezolizumab, other ICPIs are currently of interest in cancer treatment, and their role has also been investigated for UC. A Phase I trial with avelumab, an antiPDL1 antibody, was carried out in 129 patients with mUC who had progressed after PBCT or were platinum-ineligible. ${ }^{83}$ The ORR was $16 \%$, the incidence rate of grade 3/4 AEs $7 \%$, and only one death (or grade $5 \mathrm{AE}$ ) occurred, due to pneumonitis. A Phase III trial with avelumab is currently recruiting patients to evaluate its role for maintenance therapy in mUCB patients who have not progressed during or following first-line systemic therapy (NCT02603432); primary outcomes of the study are expected in July $2019 .{ }^{84}$ Durvalumab, another PDL1 antibody, was studied in a Phase I/II trial, which included 61 patients with mUC. ${ }^{85}$ The ORR was $31 \%$, with median time of response not reached. Interestingly, $46.4 \%$ of the patients with positive PDL1 expression showed response, while none of the PDL1-negative patients responded. Grade 3 AEs occurred only in $4.9 \%$ of patients, and grade 4 or 5 AEs were not seen. A Phase III trial of durvalumab is currently recruiting patients with stage IV UC to evaluate it as a monotherapy and a combined therapy with tremelimumab (CTLA4 inhibitor) versus standard-of-care chemotherapy (gemcitabine + cisplatin or gemcitabine + carboplatin) in first-line treatment, and might lead to the first first-line approval for immunotherapeutics in bladder cancer (NCT02516241). ${ }^{86}$

Nivolumab, an anti-PD1 antibody, was evaluated in 270 mUC patients who progressed or recurred following first-line systemic therapy (CheckMate-275 study). ${ }^{87}$ The ORR and median OS were 19.6\% (15\%-24.9\%) and 8.7 months, respectively. While response rates were associated with numerically higher ORRs and OS in patients with high PDL1 expression (PDL1 $\geq 5 \%: 28.4 \%$ and 11.3 months vs $16.1 \%$ and 5.95 months, respectively), patients with little or no PDL1 expression still showed ORRs of over $10 \%$ as well as durable responses. Grade 3/4 TrAEs occurred in $17.8 \%$ of patients, and three patients died from treatmentrelated pneumonitis, acute respiratory failure, and acute cardiovascular failure in this trial. As of February 2017, Nivolumab is the second FDA approved agent for treatment of mUCB after failure of PBCT.

Pembrolizumab, an anti-PD1 antibody and an approved agent for treatment of metastatic melanoma and metastatic non-small-cell lung cancer, has been tested in mUC patients resistant to, or who have progressed during, PBCT (NCT02256436/KEYNOTE-045). ${ }^{88}$ This Phase III clinical trial started on October 2014, and aimed to compare efficacy and safety of pembrolizumab $3 \mathrm{mg} / \mathrm{kg}$ every 3 weeks with investigator choice of second-line paclitaxel, docetaxel, or vinflunine, thus offering the first comparative data in this space. Recently, the trial was stopped early, since it had demonstrated superiority of pembrolizumab over chemotherapeutics and met the primary end point (improved OS) already at interim analyses. Overall, 542 patients were randomized in a 1:1 fashion to either treatment group, and the ORR for the total cohort was $21.1 \%$ without difference with respect to PDL1 expression. ${ }^{89}$ The rate of complete remissions was 7\%. While PFS was not significantly different between pembrolizumab and chemotherapy, OS was significantly different, with a hazard ratio of $0.73(0.59-0.91)$ and a median OS of 10.3 (8-11.8) vs 7.4 (6.1-8.3) months. One-year survival probability was $43.9 \%$ vs $30.7 \%$. Grade $3-5$ AEs were observed in $15 \%$ of patients for pembrolizumab vs $49.4 \%$ for the chemotherapy arm. The current velocity in the field of immunooncology in UC is highlighted by the designation of breakthrough status of durvalumab as well as subsequent fast track approval of atezolizumab and nivolumab by the FDA.

\section{Safety profile and side effects}

According to the results reported thus far in clinical trials, atezolizumab appears to be safe and highly tolerable; however, some rare immunomediated AEs (ImAEs) have been 
reported. A Phase I trial in metastatic renal cell carcinoma offered patients $10-20 \mathrm{mg} / \mathrm{kg}$ IV atezolizumab every 3 weeks, comprising a total of 16 cycles.${ }^{90}$ Neither maximum-tolerated dose was reached nor dose-limiting toxicity detected. Powles et al evaluated the safety of atezolizumab in Phase Ia expansion study of 68 patients with mUCB, of whom 93\% had received previous $\mathrm{PBCT} .{ }^{28}$ Approximately a third of patients had liver metastasis and impaired renal function, defined as a creatinine-clearance rate of less than $60 \mathrm{~mL} / \mathrm{min} / 1.73 \mathrm{~m}^{2}$. Atezolizumab was administered IV $15 \mathrm{mg} / \mathrm{kg}$ every 3 weeks for a median of 65 (range 1-259) days. Overall, 91.2\% of patients reported AEs of any grade; nevertheless $19.1 \%$ were grade 3 or 4 . Among grade 3/4 AEs, dehydration (grade 3/4, $4.4 \%$ ), cerebrovascular accident (grade $3 / 4,2.9 \%$ ), urinary tract infection (grade $3 / 4,2.9 \%$ ), and anemia (grade $3 / 4$, $2.9 \%$ ) were the most common, whereas asthenia (1.5\%), blood phosphorus decrease (1.5\%), and thrombocytopenia (1.5\%) were grade 3 AEs only. None of the patients experienced a grade 5 TrAE (or death).

Further conclusions about the safety profile of atezolizumab can be drawn from the Phase II trial. ${ }^{12}$ It evaluated the safety of atezolizumab with a fixed dose of IV 1,200 mg every 3 weeks. Due to the expectation of ImAEs, the authors reported AEs in three categories (all-cause, treatment-related, immunomediated). Any-grade all-cause AEs were reported in $97 \%$ of patients, of whom $55 \%$ had grade $3-4$ AEs. TrAEs of any grade and grade 3/4 were observed in 69\% and 16\% of patients, respectively. Among them, fatigue (any grade, $30 \%$; grade $3 / 4,2 \%$ ), nausea (any grade, $14 \%$; grade $3 / 4,0$ ), decreased appetite (any grade, 12\%; grade 3/4, 2\%), and pruritus (any grade, 10\%; grade 3/4, <1\%) were the most common. Pyrexia, diarrhea, arthralgia, vomiting, anemia, hypotension, hypertension, and colitis were less common TrAEs, with incidence of $<10 \%$ for any grade and $<1 \%$ for grade 3/4. No grade 5 TrAE was observed. ImAEs of any grade and grade $3 / 4$ were observed in $7 \%$ and $5 \%$ of patients, respectively. Rash (any grade, $7 \%$; grade $3 / 4,<1 \%$ ) was the most common ImAE; pneumonitis, dyspnea, and elevated liver enzymes (ALT and AST) were the other ImAEs, with incidence rates of $1 \%-3 \%$ for both any grade and grade $3 / 4$. Neither immunomediated nephrotoxicity nor febrile neutropenia was reported. Overall, temporary dose interruptions due to AEs were necessary for $30 \%$ of patients, and $4 \%$ of patients had to discontinue atezolizumab permanently. Up to $22 \%$ of patients were treated with systemic steroids, due to ImAEs and other AEs.

Despite overall favorable tolerability and limited follow-up, rare severe ImAEs and other AEs have been described for immunotherapies, including several fatalities. The analysis of group 1 of the IMvigor210 study, utilizing atezolizumab as a first-line therapy for mUCB patients ineligible for PBCT due to impaired renal function or ECOG score $\geq 2(n=119)$, reported one grade 5 TrAE, due to sepsis. ${ }^{45}$ In a Phase IB study of durvalumab (an anti-PDL1 antibody) in combination with tremelimumab (an anti-CTLA4 antibody) for non-small-cell lung cancer treatment, three treatmentrelated deaths were reported, due to complications arising from myasthenia gravis, pericardial effusion, and a neuromuscular disorder. ${ }^{91}$ It is important to recognize that ImAEs appear to be a class effect of these drugs, and not dose-related. Therefore, they need to be managed in a timely manner, and will not necessarily be adequately managed by dose reduction or interruption of treatment alone, but might require systemic immunosuppression.

Dose reductions are generally not recommended for atezolizumab; however, permanent discontinuation is warranted under certain circumstances ${ }^{39}$ (Table 2). No significant drug interactions have been reported. Dose adjustments are not necessary for patients with moderate renal impairment or mild hepatic impairment or for geriatric patients. Patients should be offered liver- and thyroid-function tests periodically during treatment. Monitoring patients for signs and symptoms of infections and inflammations, such as pneumonitis, meningitis, and colitis, is also recommended. Pregnant and lactating women should be warned about potential risks to fetus and infants.

The low incidence of AEs seen in anti-PDL1 agents like atezolizumab may be due to their unique mechanism of action. It leaves PDL2, the other ligand that interacts

Table 2 Adverse events that warrant permanent discontinuation of atezolizumab treatment

\begin{tabular}{ll}
\hline Adverse events & Grade (CTCAE) \\
\hline Pneumonitis & Grade 3 or 4 \\
Infusion-related reactions & Grade 3 or 4 \\
Ocular inflammatory toxicity & Grade 3 or 4 \\
Pancreatitis & Grade 4 or any grade if recurrent \\
Diarrhea or colitis & Grade 4 \\
Hypophysitis* & Grade 4 \\
Rash & Grade 4 \\
Meningoencephalitis & Any grade \\
Myasthenic syndrome/myasthenia & Any grade \\
gravis/Guillain-Barré & \\
AST or ALT $>5 \times$ ULN & Grade 3 or 4 \\
Total bilirubin $>3 \times$ ULN & Grade 3 or 4 \\
\hline
\end{tabular}

Note: *Due to probably permanent damage of the gland, experts consider continuation of atezolizumab with hormonal substitution a possible approach.

Abbreviations: CTCAE, Common Terminology Criteria for Adverse Events; ULN, upper limit of normal; AST, aspartate aminotransferase; ALT, alanine aminotransferase. 
with PD1, uninhibited, thus decreasing the incidence of severe inflammatory reactions. ${ }^{29,92}$ Ipilimumab (antibody against CTLA4), on the other hand, led to such ImAEs as hypophysitis, colitis, and vitiligo in $60 \%$ of patients overall. Grade 3/4 ImAEs were as high as $10 \%-15 \%$, with a need for high-dose corticosteroids and infliximab in refractory cases. ${ }^{93}$ More information about the safety profile of this novel antitumoral agent may be obtained from forthcoming Phase III trials.

\section{Perspective}

While PBCT remains the standard of care for advanced or mUCB, the advent of ICPIs has ushered in a new era of immunotherapy for systemic bladder cancer. With a long history of immunotherapy in the form of BCG instillations to treat non-muscle-invasive bladder cancer and a large somatic mutational burden associated with UC, mUCB seems to be an ideal candidate for treatment with ICP inhibition. Atezolizumab, an anti-PDL1 antibody, has shown efficacy as a second-line agent for patients with mUCB who progressed after PBCT, leading to FDA approval in this setting. Ongoing trials are being performed with atezolizumab in a number of different settings for UC, as are other competing ICPIs. One potential benefit of immunotherapies is that they work by a different mechanism than chemotherapy and have different toxicities. As such, there may be an opportunity for combining therapies to enhance efficacy. Also, the combination of different immunomanipulations, as seen for example in malignant melanoma, with the combination of different ICPIs to enhance immunosensitizing and outcomes is possible in bladder cancer and presents an interesting approach.

The landscape of treatment of UCB has already shifted significantly, and based on the number of active trials with different ICPIs and combination treatments with ICPIs, further manipulations of the immunooncology axis are awaited. Further elucidation of biomarkers predictive of response to systemic treatment will guide a more personalized approach to therapies, and immunotherapies will be no different from already-existing paradigms for precision medicine.

\section{Conclusion}

Atezolizumab appears to be a safe and well-tolerated secondline agent for mUCB. In a Phase II study, atezolizumab was reported to have higher response rates than historical comparisons to other second-line agents, and in some cases patients were noted to experience durable responses. These exciting findings have led to early FDA approval of atezolizumab in this setting. While large, comparative trials are lacking to date, these are under way and will further inform us about the efficacy and safety of atezolizumab for UCB. From numerous ongoing clinical trials of this agent and other ICPIs in various clinical settings, it is likely that many other options will exist for the management of UC.

\section{Disclosure}

$\mathrm{MB}$ has received honoraria for advisory boards from Genentech/Roche, Bristol-Myers Squibb, and MSD, speaking fees from Bristol-Myers Squibb, and travel compensation from Bristol-Myers Squibb. LMK has received speaking fees and travel compensation from MSD. The other authors report no conflicts of interest in this work.

\section{References}

1. Stenzl A, Cowan NC, De Santis M, et al. The updated EAU guidelines on muscle-invasive and metastatic bladder cancer. Eur Urol. 2009;55(4): 815-825.

2. Antoni S, Ferlay J, Soerjomataram I, Znaor A, Jemal A, Bray F. Bladder cancer incidence and mortality: a global overview and recent trends. Eur Urol. 2017;71(1):96-108.

3. Ploeg M, Aben KK, Hulsbergen-van de Kaa CA, Schoenberg MP, Witjes JA, Kiemeney LA. Clinical epidemiology of nonurothelial bladder cancer: analysis of the Netherlands Cancer Registry. J Urol. 2010; 183(3):915-920.

4. Mostafa MH, Sheweita SA, O’Connor PJ. Relationship between schistosomiasis and bladder cancer. Clin Microbiol Rev. 1999;12(1): 97-111.

5. Nielsen ME, Smith AB, Meyer AM, et al. Trends in stage-specific incidence rates for urothelial carcinoma of the bladder in the United States: 1988 to 2006. Cancer. 2014;120(1):86-95.

6. Andreassen BK, Aagnes B, Gislefoss R, Andreassen M, Wahlqvist R. Incidence and survival of urothelial carcinoma of the urinary bladder in Norway 1981-2014. BMC Cancer. 2016;16:799.

7. Abida W, Bajorin DF, Rosenberg JE. First-line treatment and prognostic factors of metastatic bladder cancer for platinum-eligible patients. Hematol Oncol Clin North Am. 2015;29(2):319-328, ix-x.

8. Saxman SB, Propert KJ, Einhorn LH, et al. Long-term follow-up of a phase III intergroup study of cisplatin alone or in combination with methotrexate, vinblastine, and doxorubicin in patients with metastatic urothelial carcinoma: a cooperative group study. J Clin Oncol. 1997; 15(7):2564-2569.

9. von der Maase H, Hansen SW, Roberts JT, et al. Gemcitabine and cisplatin versus methotrexate, vinblastine, doxorubicin, and cisplatin in advanced or metastatic bladder cancer: results of a large, randomized, multinational, multicenter, phase III study. J Clin Oncol. 2000;18(17): 3068-3077.

10. Bellmunt J, Fougeray R, Rosenberg JE, et al. Long-term survival results of a randomized phase III trial of vinflunine plus best supportive care versus best supportive care alone in advanced urothelial carcinoma patients after failure of platinum-based chemotherapy. Ann Oncol. 2013;24(6): 1466-1472.

11. Oing C, Rink M, Oechsle K, Seidel C, von Amsberg G, Bokemeyer C. Second line chemotherapy for advanced and metastatic urothelial carcinoma - vinflunine and beyond: a comprehensive review of the current literature. J Urol. 2016;195(2):254-263.

12. Rosenberg JE, Hoffman-Censits J, Powles T, et al. Atezolizumab in patients with locally advanced and metastatic urothelial carcinoma who have progressed following treatment with platinum-based chemotherapy: a singlearm, multicentre, phase 2 trial. Lancet. 2016;387(10031):1909-1920. 
13. Chen DS, Mellman I. Oncology meets immunology: the cancerimmunity cycle. Immunity. 2013;39(1):1-10.

14. Pardoll DM. The blockade of immune checkpoints in cancer immunotherapy. Nat Rev Cancer. 2012;12(4):252-264.

15. Zou W, Chen L. Inhibitory B7-family molecules in the tumour microenvironment. Nat Rev Immunol. 2008;8(6):467-477.

16. Zang X, Allison JP. The B7 family and cancer therapy: costimulation and coinhibition. Clin Cancer Res. 2007;13(18 Pt 1):5271-5279.

17. Krummel MF, Allison JP. CD28 and CTLA-4 have opposing effects on the response of T cells to stimulation. J Exp Med. 1995;182(2):459-465.

18. Hodi FS, O'Day SJ, McDermott DF, et al. Improved survival with ipilimumab in patients with metastatic melanoma. N Engl J Med. 2010; 363(8):711-723.

19. Bertrand A, Kostine M, Barnetche T, Truchetet ME, Schaeverbeke T. Immune related adverse events associated with anti-CTLA-4 antibodies: systematic review and meta-analysis. BMC Med. 2015;13:211.

20. Ohaegbulam KC, Assal A, Lazar-Molnar E, Yao Y, Zang X. Human cancer immunotherapy with antibodies to the PD-1 and PD-L1 pathway. Trends Mol Med. 2015;21(1):24-33.

21. Dong H, Strome SE, Salomao DR, et al. Tumor-associated B7-H1 promotes T-cell apoptosis: a potential mechanism of immune evasion. Nat Med. 2002;8(8):793-800.

22. Flies DB, Chen L. The new B7s: playing a pivotal role in tumor immunity. J Immunother. 2007;30(3):251-260.

23. Berger R, Rotem-Yehudar R, Slama G, et al. Phase I safety and pharmacokinetic study of CT-011, a humanized antibody interacting with PD-1, in patients with advanced hematologic malignancies. Clin Cancer Res. 2008;14(10):3044-3051.

24. Patnaik A, Kang SP, Rasco D, et al. Phase I study of pembrolizumab (MK-3475; anti-PD-1 monoclonal antibody) in patients with advanced solid tumors. Clin Cancer Res. 2015;21(19):4286-4293.

25. Wong RM, Scotland RR, Lau RL, et al. Programmed death-1 blockade enhances expansion and functional capacity of human melanoma antigen-specific CTLs. Int Immunol. 2007;19(10):1223-1234.

26. Motzer RJ, Escudier B, McDermott DF, et al. Nivolumab versus everolimus in advanced renal-cell carcinoma. N Engl J Med. 2015;373(19): 1803-1813.

27. Bidnur S, Savdie R, Black PC. Inhibiting immune checkpoints for the treatment of bladder cancer. Bladder Cancer. 2016;2(1):15-25.

28. Powles T, Eder JP, Fine GD, et al. MPDL3280A (anti-PD-L1) treatment leads to clinical activity in metastatic bladder cancer. Nature. 2014; 515(7528):558-562.

29. Brahmer JR, Tykodi SS, Chow LQ, et al. Safety and activity of antiPD-L1 antibody in patients with advanced cancer. N Engl J Med. 2012; 366(26):2455-2465.

30. Beck A, Wurch T, Reichert JM. 6th Annual European Antibody Congress 2010: November 29-December 1, 2010, Geneva, Switzerland. mAbs. 2011;3(2):111-132.

31. Boyerinas B, Jochems C, Fantini M, et al. Antibody-dependent cellular cytotoxicity activity of a novel anti-PD-L1 antibody avelumab (MSB0010718C) on human tumor cells. Cancer Immunol Res. 2015; 3(10):1148-1157.

32. Morales A, Eidinger D, Bruce AW. Intracavitary bacillus Calmette-Guérin in the treatment of superficial bladder tumors. J Urol. 1976;116(2): $180-183$.

33. Donin NM, Lenis AT, Holden S, et al. Immunotherapy in the treatment of urothelial carcinoma. J Urol. 2017;197(1):14-22.

34. Redelman-Sidi G, Glickman MS, Bochner BH. The mechanism of action of BCG therapy for bladder cancer: a current perspective. Nat Rev Urol. 2014;11(3):153-162.

35. Inman BA, Sebo TJ, Frigola X, et al. PD-L1 (B7-H1) expression by urothelial carcinoma of the bladder and BCG-induced granulomata: associations with localized stage progression. Cancer. 2007;109(8): 1499-1505

36. Cancer Genome Atlas Research Network. Comprehensive molecular characterization of urothelial bladder carcinoma. Nature. 2014;507(7492): 315-322.
37. Herbst RS, Soria JC, Kowanetz M, et al. Predictive correlates of response to the anti-PD-L1 antibody MPDL3280A in cancer patients. Nature. 2014;515(7528):563-567.

38. Park JJ, Omiya R, Matsumura Y, et al. B7-H1/CD80 interaction is required for the induction and maintenance of peripheral T-cell tolerance. Blood. 2010;116(8):1291-1298.

39. Tecentriq (atezolizumab) injection for intravenous use [prescribing information]. San Francisco: Genentech; 2016.

40. Deng R, Bumbaca D, Pastuskovas CV, et al. Preclinical pharmacokinetics, pharmacodynamics, tissue distribution, and tumor penetration of anti-PD-L1 monoclonal antibody, an immune checkpoint inhibitor. mAbs. 2016;8(3):593-603.

41. Eisenhauer EA, Therasse P, Bogaerts J, et al. New response evaluation criteria in solid tumours: revised RECIST guideline (version 1.1). Eur J Cancer. 2009;45(2):228-247.

42. Wolchok JD, Hoos A, O'Day S, et al. Guidelines for the evaluation of immune therapy activity in solid tumors: immune-related response criteria. Clin Cancer Res. 2009;15(23):7412-7420.

43. Hoffmann-La Roche. A study of atezolizumab in patients with locally advanced or metastatic urothelial bladder cancer [IMvigor210]. Available from: https://clinicaltrials.gov/ct2/show/NCT02108652. NLM identifier: NCT02108652. Accessed December 6, 2016.

44. Loriot Y, Rosenberg JE, Powles TB, et al. Atezolizumab (atezo) in platinum (plat)-treated locally advanced/metastatic urothelial carcinoma (mUC): updated OS, safety and biomarkers from the Ph II IMvigor210 study. Ann Oncol. 2016;27(6):266-295.

45. Bellmunt J, Balar A, Galsky MD, et al. IMvigor210: updated analyses of first-line (1L) atezolizumab (atezo) in cisplatin (cis)-ineligible locally advanced/metastatic urothelial carcinoma (mUC). Ann Oncol. 2016;27(6):266-295.

46. Hoffmann-La Roche. A study of atezolizumab in participants with locally advanced or metastatic urothelial bladder cancer (cohort 1). Available from: https://clinicaltrials.gov/ct2/show/NCT02951767. NLM identifier: NCT02951767. Accessed December 6, 2016.

47. Bellmunt J, Choueiri TK, Schutz FA, Rosenberg JE. Randomized phase III trials of second-line chemotherapy in patients with advanced bladder cancer: progress and pitfalls. Ann Oncol. 2011;22(2): 245-247.

48. Hoffmann-La Roche. A study of atezolizumab compared with chemotherapy in participants with locally advanced or metastatic urothelial bladder cancer [IMvigor211]. Available from: https://clinicaltrials. gov/ct2/show/record/NCT02302807. NLM identifier: NCT02302807. Accessed November 10, 2016.

49. Hoffmann-La Roche. The effect of atezolizumab in combination with gemcitabine/carboplatin and gemcitabine/carboplatin alone in participants with untreated locally advanced or metastatic urothelial carcinoma who are ineligible for cisplatin-based therapy [IMvigor130]. Available from: https:/clinicaltrials.gov/ct2/show/NCT02807636. NLM identifier: NCT02807636. Accessed November 10, 2016

50. Hoffmann-La Roche. A phase III study of atezolizumab treatment versus observation as adjuvant therapy in patients with PD-L1 positive, high risk muscle invasive bladder cancer after cystectomy [IMvigor010]. Available from: https://clinicaltrials.gov/ct2/show/NCT02450331. NLM identifier: NCT02450331. Accessed November 10, 2016.

51. Hoffmann-La Roche. Safety and pharmacology study of atezolizumab alone and in combination with bacille Calmette-Guérin (BCG) in high-risk non-muscle-invasive bladder cancer (NMIBC) participants. Available from: https:/clinicaltrials.gov/ct2/show/record/NCT02792192. NLM identifier: NCT02792192. Accessed November 15, 2016.

52. Queen Mary University of London. Preoperative MPDL3280A in transitional cell carcinoma of the bladder (ABACUS). Available from: https:/clinicaltrials.gov/ct2/show/record/NCT02662309. NLM identifier: NCT02662309. Accessed November 15, 2016.

53. University of California, San Francisco. Study of MPDL3280A in bladder cancer. Available from: https://clinicaltrials.gov/ct2/ show/NCT02451423. NLM identifier: NCT02451423. Accessed November 15, 2016. 
54. National Cancer Institute (NCI). Atezolizumab in treating patients with recurrent BCG-unresponsive non-muscle invasive bladder cancer. Available from: https://clinicaltrials.gov/ct2/show/NCT02844816. NLM identifier: NCT02844816. Accessed November 15, 2016.

55. Corvus Pharmaceuticals. Phase 1/1B study to evaluate the safety and tolerability of CPI-444 alone and in combination with atezolizumab in advanced cancers. Available from: https://clinicaltrials.gov/ct2/show/ record/NCT02655822. NLM identifier: NCT02655822. Accessed November 15, 2016.

56. Celldex Therapeutics. A study of varlilumab and atezolizumab in patients with advanced cancer. Available from: https://clinicaltrials. gov/ct2/show/record/NCT02543645. NLM identifier: NCT02543645. Accessed November 15, 2016.

57. Genentech. A study of atezolizumab administered in combination with bevacizumab and/or with chemotherapy in participants with locally advanced or metastatic solid tumors. Available from: https://clinicaltrials.gov/ct2/show/NCT01633970. NLM identifier: NCT01633970. Accessed November 15, 2016.

58. Incyte Corporation. A study of atezolizumab (MPDL3280A) in combination with epacadostat (INCB024360) in subjects with previously treated stage IIIB or stage IV non-small cell lung cancer and previously treated stage IV urothelial carcinoma (ECHO-110). Available from https://clinicaltrials.gov/ct2/show/NCT02298153. NLM identifier: NCT02298153. Accessed November 20, 2016.

59. Bellesoeur A, Massard C, Soria JC. Biomarkers in cancer immunotherapy: analysis of clinical, histological and immunohistochemical factors associated with PD-L1 status. Ann Oncol. 2016;27 Suppl 6:82P.

60. Taube JM, Klein A, Brahmer JR, et al. Association of PD-1, PD-1 ligands, and other features of the tumor immune microenvironment with response to anti-PD-1 therapy. Clin Cancer Res. 2014;20(19):5064-5074.

61. Gainor JF, Shaw AT, Sequist LV, et al. EGFR mutations and ALK rearrangements are associated with low response rates to PD-1 pathway blockade in non-small cell lung cancer: a retrospective analysis Clin Cancer Res. 2016;22(18):4585-4593.

62. Madore J, Vilain RE, Menzies AM, et al. PD-L1 expression in melanoma shows marked heterogeneity within and between patients: implications for anti-PD-1/PD-L1 clinical trials. Pigment Cell Melanoma Res. 2015; 28(3):245-253.

63. Callea M, Albiges L, Gupta M, et al. Differential expression of PD-L1 between primary and metastatic sites in clear-cell renal cell carcinoma Cancer Immunol Res. 2015;3(10):1158-1164.

64. Anantharaman A, Friedlander T, Lu D, et al. Programmed deathligand 1 (PD-L1) characterization of circulating tumor cells (CTCs) in muscle invasive and metastatic bladder cancer patients. BMC Cancer. 2016;16:744.

65. Schehr JL, Schultz ZD, Warrick JW, et al. High specificity in circulating tumor cell identification is required for accurate evaluation of programmed death-ligand 1. PLoS One. 2016;11(7):e0159397.

66. Sonpavde G, Pond GR, Mullane S, et al. Outcomes in patients with advanced urothelial carcinoma after discontinuation of programmed death (PD)-1 or PD ligand 1 inhibitor therapy. BJU Int. Epub 2016 Oct 4.

67. Bellmunt J, Theodore C, Demkov T, et al. Phase III trial of vinflunine plus best supportive care compared with best supportive care alone after a platinum-containing regimen in patients with advanced transitional cell carcinoma of the urothelial tract. J Clin Oncol. 2009;27(27): 4454-4461.

68. Logothetis CJ, Dexeus FH, Sella A, et al. Escalated therapy for refractory urothelial tumors: methotrexate-vinblastine-doxorubicin-cisplatin plus unglycosylated recombinant human granulocyte-macrophage colony-stimulating factor. J Natl Cancer Inst. 1990;82(8):667-672.

69. Kattan J, Culine S, Theodore C, Droz JP. Second-line M-VAC therapy in patients previously treated with the M-VAC regimen for metastatic urothelial cancer. Ann Oncol. 1993;4(9):793-794.

70. Kim YS, Lee SI, Park SH, et al. A phase II study of weekly docetaxel as second-line chemotherapy in patients with metastatic urothelial carcinoma. Clin Genitourin Cancer. 2016;14(1):76-81.
71. Albers $\mathrm{P}$, Siener R, Härtlein $\mathrm{M}$, et al. Gemcitabine monotherapy as second-line treatment in cisplatin-refractory transitional cell carcinoma: prognostic factors for response and improvement of quality of life. Onkologie. 2002;25(1):47-52.

72. Witte RS, Elson P, Bono B, et al. Eastern Cooperative Oncology Group phase II trial of ifosfamide in the treatment of previously treated advanced urothelial carcinoma. J Clin Oncol. 1997;15(2):589-593.

73. Logothetis CJ, Hossan E, Sella A, Dexeus FH, Amato RJ. Fluorouracil and recombinant human interferon alfa-2a in the treatment of metastatic chemotherapy-refractory urothelial tumors. $J$ Natl Cancer Inst. 1991; 83(4):285-288.

74. Vaughn DJ, Broome CM, Hussain M, Gutheil JC, Markowitz AB. Phase II trial of weekly paclitaxel in patients with previously treated advanced urothelial cancer. J Clin Oncol. 2002;20(4):937-940.

75. Sweeney CJ, Roth BJ, Kabbinavar FF, et al. Phase II study of pemetrexed for second-line treatment of transitional cell cancer of the urothelium. J Clin Oncol. 2006;24(21):3451-3457.

76. Ortmann CA, Mazhar D. Second-line systemic therapy for metastatic urothelial carcinoma of the bladder. Future Oncol. 2013;9(11): $1637-1651$

77. Dreicer R, Li H, Stein M, et al. Phase 2 trial of sorafenib in patients with advanced urothelial cancer: a trial of the Eastern Cooperative Oncology Group. Cancer. 2009;115(18):4090-4095.

78. Twardowski P, Stadler WM, Frankel P, et al. Phase II study of aflibercept (VEGF-Trap) in patients with recurrent or metastatic urothelial cancer, a California Cancer Consortium trial. Urology. 2010;76(4): 923-926.

79. Necchi A, Mariani L, Zaffaroni N, et al. Pazopanib in advanced and platinum-resistant urothelial cancer: an open-label, single group, phase 2 trial. Lancet Oncol. 2012;13(8):810-816.

80. Niegisch G, Retz M, Thalgott M, et al. Second-line treatment of advanced urothelial cancer with paclitaxel and everolimus in a German phase II trial (AUO trial AB 35/09). Oncology. 2015;89(2): 70-78.

81. Witjes JA, Comperat E, Cowan NC, et al. EAU guidelines on muscleinvasive and metastatic bladder cancer: summary of the 2013 guidelines. Eur Urol. 2014;65(4):778-792.

82. Albers P, Park SI, Niegisch G, et al. Randomized phase III trial of 2nd line gemcitabine and paclitaxel chemotherapy in patients with advanced bladder cancer: short-term versus prolonged treatment (German Association of Urological Oncology [AUO] trial AB 20/99). Ann Oncol. 2011; 22(2):288-294.

83. Patel MR, Ellerton J, Agrawal M, et al. Avelumab (MSB0010718C; anti-PD-L1) in patients with metastatic urothelial carcinoma progressed after platinum-based therapy or platinum ineligible. Ann Oncol. 2016; 27 Suppl 6:777PD.

84. Pfizer. A Study of avelumab in patients with locally advanced or metastatic urothelial cancer (JAVELIN Bladder 100). Available from: https://clinicaltrials.gov/ct2/show/record/NCT02603432. NLM identifier: NCT02603432. Accessed November 19, 2016.

85. Massard C, Gordon MS, Sharma S, et al. Safety and efficacy of durvalumab (MEDI4736), an anti-programmed cell death ligand-1 immune checkpoint inhibitor, in patients with advanced urothelial bladder cancer. J Clin Oncol. 2016;34(26):3119-3125.

86. AstraZeneca. Study of MEDI4736 with or without tremelimumab versus standard of care chemotherapy in urothelial cancer. Available from: https://clinicaltrials.gov/ct2/show/NCT02516241. NLM identifier: NCT02516241. Accessed December 6, 2016.

87. Galsky MD, Retz M, Siefker-Radtke AO, et al. Efficacy and safety of nivolumab monotherapy in patients with metastatic urothelial cancer (mUC) who have received prior treatment: results from the phase II CheckMate 275 study. Ann Oncol. 2016;27 Suppl 6:LBA31.

88. Merck. A study of pembrolizumab (MK-3475) versus paclitaxel, docetaxel, or vinflunine for participants with advanced urothelial cancer (MK-3475-045/KEYNOTE-045). Available from: https://clinicaltrials. gov/ct2/show/study/NCT02256436. NLM identifier: NCT02256436. Accessed November 19, 2016. 
89. Bellmunt J, de Wit R, Vaughn DJ, et al. Open-label, phase III study of pembrolizumab versus investigator's choice of paclitaxel, docetaxel, or vinflunine for previously treated advanced urothelial cancer. Poster presented at: 2016 SITC Annual Meeting; November 9-13, 2016; National Harbor, MD.

90. McDermott DF, Sosman JA, Sznol M, et al. Atezolizumab, an antiprogrammed death-ligand 1 antibody, in metastatic renal cell carcinoma: long-term safety, clinical activity, and immune correlates from a phase IA study. J Clin Oncol. 2016;34(8):833-842.

91. Antonia S, Goldberg SB, Balmanoukian A, et al. Safety and antitumour activity of durvalumab plus tremelimumab in non-small cell lung cancer: a multicentre, phase 1B study. Lancet Oncol. 2016;17(3):299-308.
92. Chen DS, Irving BA, Hodi FS. Molecular pathways - next-generation immunotherapy: inhibiting programmed death-ligand 1 and programmed death-1. Clin Cancer Res. 2012;18(24):6580-6587.

93. Fong L, Small EJ. Anti-cytotoxic T-lymphocyte antigen-4 antibody: the first in an emerging class of immunomodulatory antibodies for cancer treatment. J Clin Oncol. 2008;26(32):5275-5283.

94. Meng X, Huang Z, Teng F, Xing L, Yu J. Predictive biomarkers in PD-1/PD-L1 checkpoint blockade immunotherapy. Cancer Treat Rev. 2015;41(10):868-876.

\section{Publish your work in this journal}

OncoTargets and Therapy is an international, peer-reviewed, open access journal focusing on the pathological basis of all cancers, potential targets for therapy and treatment protocols employed to improve the management of cancer patients. The journal also focuses on the impact of management programs and new therapeutic agents and protocols on

\section{Dovepress}

patient perspectives such as quality of life, adherence and satisfaction The manuscript management system is completely online and includes a very quick and fair peer-review system, which is all easy to use. Visit http://www.dovepress.com/testimonials.php to read real quotes from published authors. 